\title{
Numerical modelling of hydraulically bonded mixture with rubber admixture due to applied mechanical loadings
}

\author{
Daniel Pietras, Tomasz Sadowski \\ Department of Solid Mechanics, Faculty of Civil Engineering and Architecture, \\ University of Technology in Lublin, \\ e-mail:d.pietras@pollub.pl,e-mail:t.sadowski@pollub.pl
}

\begin{abstract}
In this paper the application of the Extended Finite Element Method (XFEM) to anticipate change of the behaviour of composite with complex internal structure after use of the admixtures was presented. The response to mechanical loadings of bent beams made of hydraulically bonded mixture with rubber admixture was considered. The impact of the rubber granulate on the value of fracture energy was analysed. Moreover, the influence of an interfacial transmission zone quality on the achieved effect was assessed. Calculations were conducted by means of the muli-scale numerical model which was built in ABAQUS finite element method environment. The results derived indicate beneficial effect of rubber granulate on fracture energy of hydraulically bonded mixture.
\end{abstract}

Keywords: Virtual test, XFEM, FEM, multi-scale modelling.

\section{Introduction}

The Hydraulically Bonded Mixture (HBM) is the material with complex internal structure. The filling effect of rubber granulate is influenced by many factors. The most important of them are:

- the mechanical properties of used rubber [1],

- the elastic properties and toughness of interfacial transmission zones (ITZ) between the rubber and cement matrix [2],

- the type of composite matrix [3],

- the shape of grains of used admixture or additive [4].

Laboratory tests are one of the ways to analyse the effect of the used additive on the apparent properties of the composite. Studying the correlation between the individual properties of the components and achieved equivalent properties of composite requires conducting many tests and to employs large amounts of often costly specimens. The time spent on laboratory tests and their cost can be reduced by developing virtual testing techniques. The virtual test can be conducted using the multi-scale numerical models which are able to reflect the real internal structure of the composite [5], [6]. Contemporary research techniques such as microtomography [7], scanning microscopy, mercury porosimetry and microhardness tests [8] allow to build accurate numerical models of concrete with internal structure mapping. Thanks to the use of material numerical models it is possible to find the relationship between the apparent properties of composite and e.g. shape of used components as well as to explain some processes such as the effect of porosity on water permeability [9]. The virtual tests can help to determine the impact of the particular ingredients and they can be essential in taking the right path in designing process of cement-matrix composites. The results derived in this way will allow narrowing down the range of the necessary 
experimental tests. Therefore, the example of simple multi-scale model of HBM was presented in this paper. This model allows assessing the change of apparent properties of HBM after application of rubber fine admixtures.

\section{Mechanical properties of HBM constituents}

\subsection{Elastic properties of rubber}

In this paper the hyperelastic model (Monney-Rivlin) of rubber in which used linear relationships of strain tensor invariants $I_{1}$ and $I_{2}$ was assumed. The strain energy potential $U$ can be expressed as following:

$$
U=C_{10}\left(I_{1}-3\right)+C_{01}\left(I_{2}-3\right)+\frac{1}{D_{1}}\left(J_{e l}-1\right)^{2}
$$

The values of material constants $C_{10}=8.601$ and $C_{01}=1.805$ are based on [10], thus the incompressibility of rubber was assumed as following $D_{1}=0 . J_{e l}$ is the ratio between the total volumetric strain and the volumetric strain caused by temperature changes.

\subsection{Properties of rubber-cement matrix interfacial zones}

The rubber-cement contact properties were analysed in [11]. The relation between cohesive properties of the rubber-cement matrix interfacial layer and the critical force which lead to splitting the rubber-cement coupling is a complex issue. The discrepancies between the obtained destructive force and the force calculated on the basis of classical fracture mechanics are high due to the high incompatibility of elastic properties [12]. Therefore the test results presented in the [11] do not allow for easy determination of the correct toughness or critical stress intensity factors of rubber-cement interfacial zones for numerical modeling. For this reason the auxiliary numerical model was built. This model, shown in Fig. 1 reflects the conditions of laboratory tests conducted in [11]. It has been used to assess proper characteristics of cement-rubber ITZ that can be applied in meso-scale numerical model of $\mathrm{HBM}$ with rubber admixture $(\mathrm{HBM}+\mathrm{G})$.

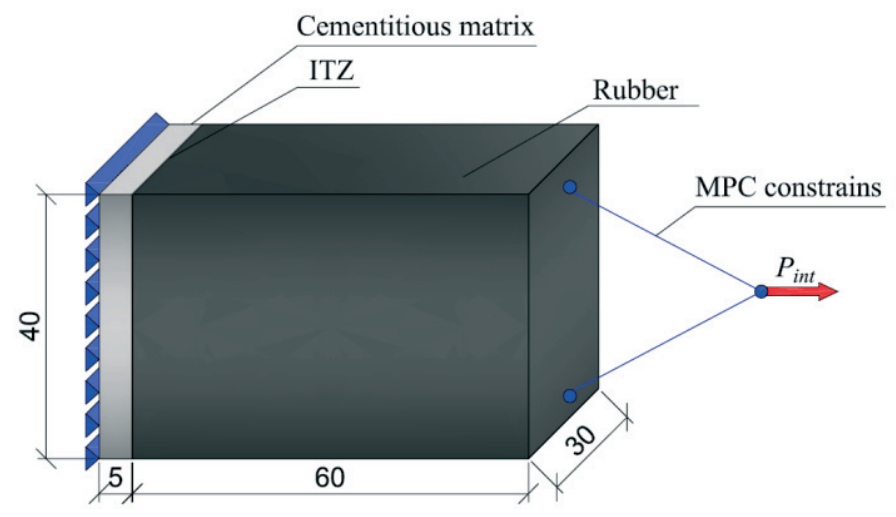

Fig. 1. Geometry and boundary conditions of auxiliary cement-rubber coupling numerical model

In presented auxiliary model the initiation of crack is determined by maximal principal stress criterion. The crack propagation process is described by linear cohesive law. In the case where the rubber-cement contact was the strongest in [11] the critical force 
achieved value $P_{\text {int }}=286.8 \mathrm{~N}$. In the numerical model the closest value $P_{\text {int }}=283.417 \mathrm{~N}$ was derived for critical stress equalled $0.9099 \mathrm{MPa}$. The value of fracture energy was assumed $0.02 \mathrm{~N} / \mathrm{m}$ so as to reflect the brittle mode of damage of considered contact.

\subsection{Composite matrix properties}

In this paper the internal structure of composite matrix has been simplified. It was modelled as isotropic body with pores. The pores radius was equal to $0.7 \mathrm{~mm}$. The preliminary laboratory tests of HBM has determined apparent modulus of elasticity $E_{\text {eq }}=15.9 \mathrm{GPa}$ and porosity about $12 \%$. To determine the matrix properties, which are necessary for the multi-scale model of HBM the auxiliary model was built (see Fig. 2). The model thickness was assumed $10 \mathrm{~mm}$ while the distance between points $\mathrm{G}$ and $\mathrm{D}$ was equal to $32.542 \mathrm{~mm}$.

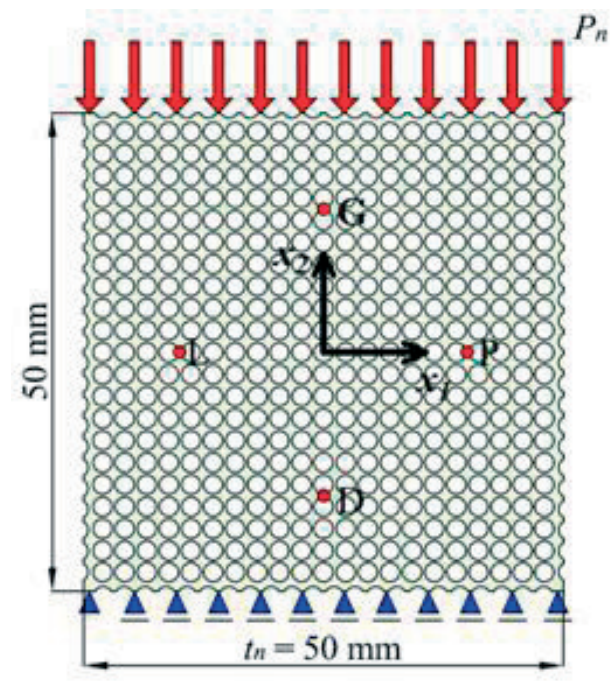

Fig. 2. The auxiliary model to determine the HBM matrix properties

By using this model the compression virtual tests were performed. The modulus of elasticity of matrix was tuned so that the apparent modulus of elasticity $E_{a p}$ of the material determined by following equation were equal to $E_{\text {eq }}$ :

$$
E_{a p}=\frac{\left(\frac{P_{n}}{500 \mathrm{~mm}^{2}}\right)}{\left(\frac{U_{G}-U_{D}}{32.542 \mathrm{~mm}}\right)}=15.9 \mathrm{MPa}
$$

According to Fig. $2 U_{G}$ and $U_{D}$ are displacements of point $\mathrm{G}$ and $\mathrm{D}$. The obtained property of the matrix modulus of elasticity was equal to $E_{m}=22.5 \mathrm{GPa}$. Poisson ratio of the matrix was assumed to be equal to 0.29 .

\subsection{Geometry of multi-scale model}

The geometry and structure of multi-scale numerical model of HBM is shown in Fig. 3. The thickness of beam was assumed to be equal to $30 \mathrm{~mm}$. Beam span to beam height ratio was maintained as recommended in crack resistance tests [13]. 


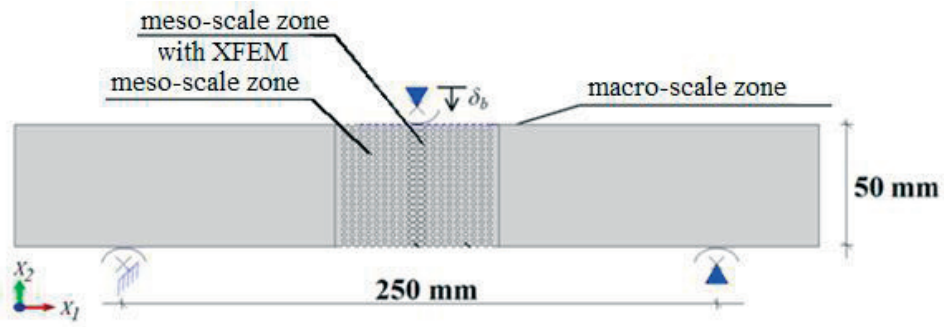

Fig. 3. The HBM beams multi-scale numerical model structure

In order to reduce the computational time, the model was divvied into three main areas where different scales of material description were used. The macro-scale zone was modelled as linear elastic and isotropic area with properties assumed on the basis of preliminary laboratory tests $E=15.9 \mathrm{GPa}, v=0.29$. The meso-scale zone was modelled according to point 2.3. Moreover in the cases where the pores were filled by rubber the ITZ zones with thickness $0.1 \mathrm{~mm}$ were modelled. They have reduced elastic properties with respect to matrix of composite due to the local increase of water to cement ratio. It results in the increased porosity of hardened cement paste in ITZ and the decrease of mechanical properties. The modulus of elasticity in ITZ zones was assumed $E_{I T Z}=15 \mathrm{GPa}$ and Poisson ratio $v_{I T Z}=0.3$. In the meso-scale zones where the cracking is impossible all constituents have assigned elastic mechanical properties. The mesh of finite elements in these zones is shown in Fig. 4A.

A)

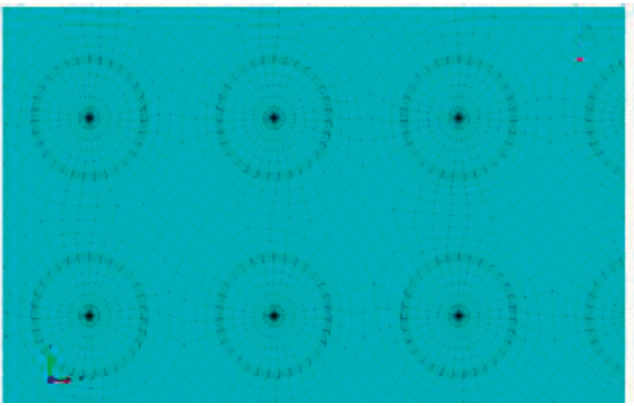

B)

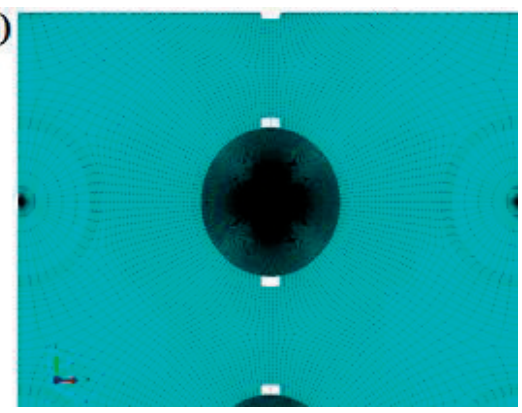

Fig. 4. A) The mesh of finite elements in regions far from expected cracking B) The mesh of finite elements in regions near expected cracking

The detailed construction of the model containing rubber crumbs in the zone near expected fracture is presented in Fig. 5. The ITZ zone has the discontinuities as real ITZ so as to simplify the calculation process. In each of the emptiness the initial crack with length $0.2 \mathrm{~mm}$ was modelled to reflect real imperfections in the material. 


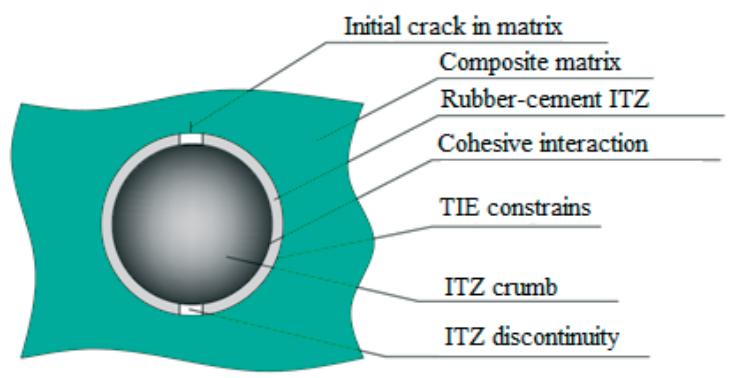

Fig. 5. Detailed construction of the single rubber crumb with ITZ layer in HBM matrix and initial cracks

The left semicircle of ITZ with matrix and rubber coupling was realized using TIE constraints which link all degrees of freedom of the corresponding nodes. The right semicircle of ITZ and matrix coupling were modelled in the same way. The right semicircle of ITZ with rubber coupling has the cohesive properties according to point 2.2. The nodes of ITZ belonging to the contact were set as MASTER and corresponding nodes of rubber as SLAVE due to this fact the mesh of rubber part is four times denser than mesh of ITZ (see Fig. 4B).

The length of initial crack in the beam was assumed close to $1 / 3$ of the beam height (Fig. 6). The pores crossed by initial crack were not filled by rubber crumbs. The initial crack of the beam was modelled by duplicating the nodes lying on its path as it is presented by thick line in Fig. 6.

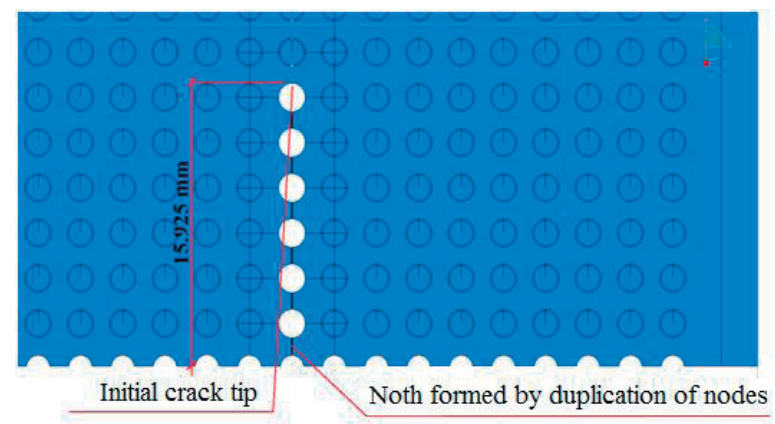

Fig. 6. The initial crack in the considered beams

The matrix cracking and ITZ is determined by maximal principal stress criterion, the angle of crack propagation is determined by maximal tangential stress [MTS] criterion. The effect of cohesive zone in concrete is taken into account by linear cohesive law. In these model the critical stress of the matrix was assumed as following $\sigma_{I c}^{\text {mat }}=1.8 \mathrm{MPa}$, the fracture energy was assumed $G=3 \mathrm{~N} / \mathrm{m}$. Values of fracture parameters are close to real properties of HBM but have exemplary character.

\section{Results of conducted analyses}

In present paper 3 cases were considered:

- HBM without rubber additives,

- HBM with rubber admixture (HBM+R),

- HBM with rubber admixture, without ITZ zones. 
The filling effect of pores by rubber particles was discussed in [14]. The Fig. 7 presents the maximal principal stress distribution in the fragment of the considered beam during the cracking process. The huge difference of the elastic properties between ITZ and matrix makes the ITZ slightly loaded. The damage of ITZ-rubber coupling takes place after damage of the particular piece of matrix due to the high deformability of rubber which is a beneficial phenomenon. This fact also coincides with the observation made during experimental tests.

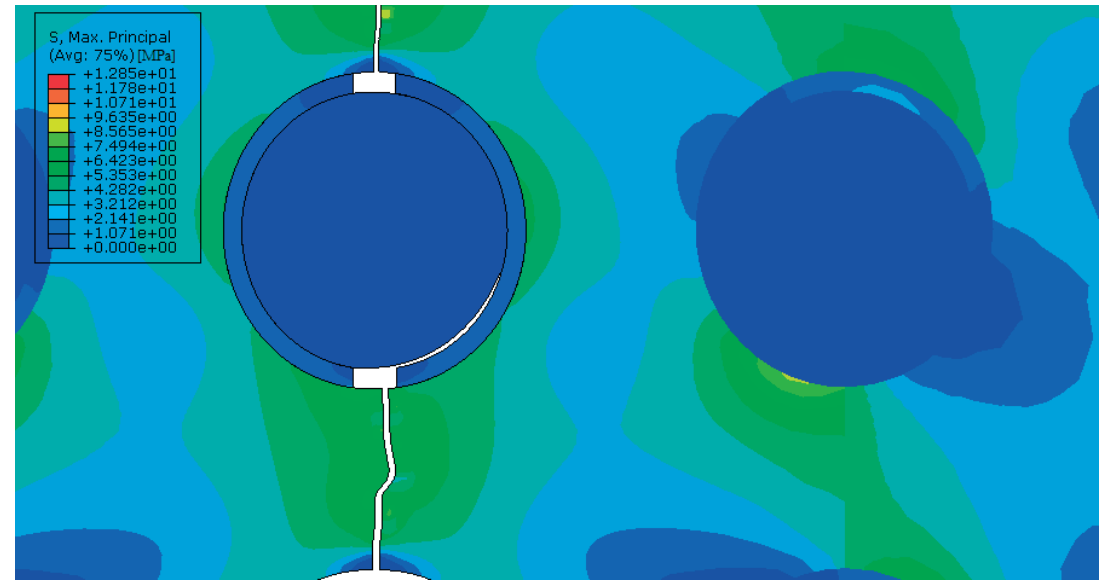

Fig. 7. ITZ-Rubber coupling damage process

The relation of loading force $P_{b}$ to middle span point displacement $\delta_{b}$ derived for beam made of $\mathrm{HBM}+\mathrm{R}$ is shown in Fig. 8. For comparison the same relation for beam made of HBM was included. Both beams indicate the same response to applied loadings in linear elastic range. Differences appear when the critical value of the loading force $P_{b}^{\max }$ was reached. In the case of beams with pores filled in with rubber $P_{b}^{\max }$ reaches slightly higher value. At further stages of loadings the differences are clear. In the beams made of $\mathrm{HBM}+\mathrm{G}$ the forces remain at a higher level.

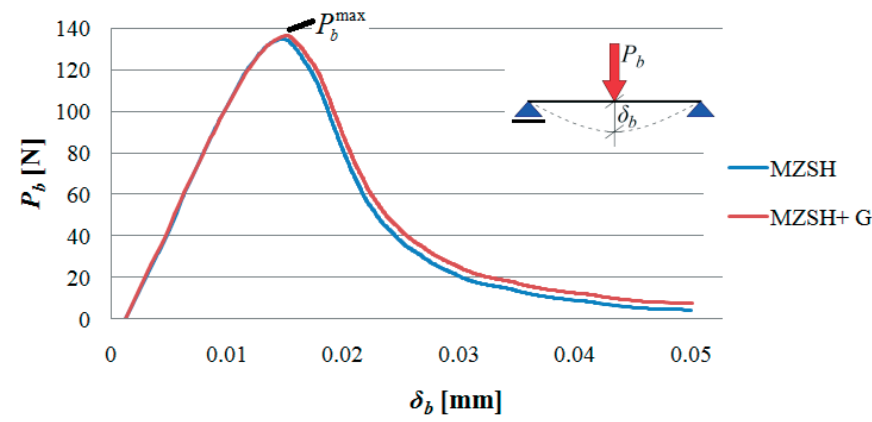

Fig. 8. Comparison of the mechanical response for beams made of $\mathrm{HBM}$ and $\mathrm{HBM}+\mathrm{G}$ in 3-point bending conditions

The analysis of bending beam made of $\mathrm{HBM}+\mathrm{G}$ without ITZ was conducted to demonstrate the effect of ITZ quality on the beam response. In order to avoid the numerical errors caused by using different finite element meshes the numerical model was modified 
only by setting as equal to $E_{m}$. In meso-scale (Fig. 3 ) these parameters were equal to properties of the composite matrix. In the present case the noticeable increase of the force used to destroy the beam can be observed. However, the post-destructive response remains unchanged as shown in Fig. 9.

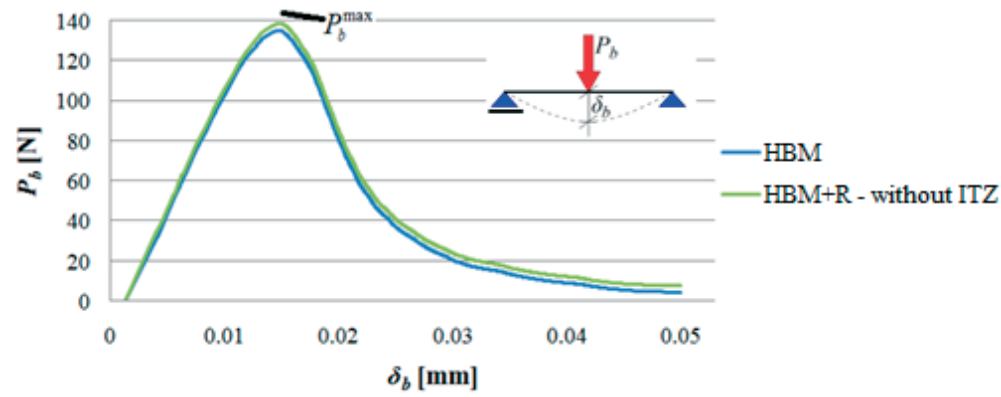

Fig. 9. Mechanical response comparison for beams made of $\mathrm{HBM}$ and $\mathrm{HBM}+\mathrm{G}$ without ITZ in 3-point bending conditions

In table 1 the results of conducted virtual bending tests were collected. The relative changes of critical forces $P_{b}^{\max }$ and fracture energy $G$ in considered cases were calculated as following:

$$
\Delta_{p}=\frac{P_{M Z S H}^{M a x}-P_{2}^{M a x}}{P_{M Z S H}^{M a x}} \cdot 100, \quad \Delta_{G}=\frac{G_{M Z S H}-G_{2}}{G_{M Z S H}} \cdot 100
$$

Where HBM subscript means properties of pure HBM and 2 subscript means a property for one of the considered cases of HBM with rubber admixture. For $\mathrm{HBM}+\mathrm{G}$ the $1 \%$ growth of $P_{b}^{\max }$ and the fracture energy increase of $2.3 \%$ with respect to pure HBM were achieved. Better results were obtained by taking into account the ideal properties of ITZ zones i.e. $2.6 \%$ increase of the $P_{b}^{\max }$ and $3.7 \%$ increase in fracture energy with respect to pure HBM.

The fracture energy $G$ was determined as the area under $P_{b}\left(\delta_{b}\right)$ relation divided into the cross section area split during the test:

$$
G_{u}=\frac{1}{30 m m \cdot(50 m m-15.925 m m)} \int_{0}^{0.005 m m} P_{b} d \delta_{b}
$$

Table 1. Results of conducted virtual tests

\begin{tabular}{ccccc}
\hline Material & $P_{b}^{\max }[\mathrm{N}]$ & $G_{u}[\mathrm{~N} / \mathrm{m}]$ & $\Delta_{p}[\%]$ & $\Delta_{G}[\%]$ \\
\hline HBM & 134.982 & 2.096 & - & - \\
HBM+R & 136.266 & 2.144 & 0.951 & 2.290 \\
HBM+R (perfect ITZ) & 138.528 & 2.174 & 2.627 & 3.721 \\
\hline
\end{tabular}

The results derived in discussed meso-scale model confirm that by using rubber crumbs admixture the fracture energy of HBM can be increased. 


\section{Summary and conclusions}

In present paper the meso-scale model of hydraulically bonded mixture was presented. The effect of filling pores in this mixture by rubber crumbs was analysed. The virtual test conducted by means of the meso-scale numerical model showed the increase of critical force by $1 \%$ and the increase of the fracture energy by $2.3 \%$. Moreover, the effect of interfacial transmission zone quality on achieved results was analysed. In case of good properties of the ITZ the increase of critical force by $2.6 \%$ and the increase of the fracture energy by $3.7 \%$ were achieved. These observations lead to the conclusion that the interfacial transmission zones between the rubber and cement matrix are of great significance in apparent properties of composite. Thus, this fact should be taken into account when designing the hydraulically bounded mixtures with rubber additives.

\section{References}

1. Benazzouk A., Mezreb K., Doyen G. , Goullieux A., Quéneudec M. Effect of rubber aggregates on the physico-mechanical behaviour of cement-rubber composites of the alveolar texture of rubber aggregates. Cement \&Concrete Composites 25 (2003) 711-720.

2. Emiroglu M., Kelestemur M.H., Yildiz S. An investigation on itz microstructure of the concrete containing waste vehicle tire. Proc of $8^{\text {th }}$ International Fracture Conference 7-8 November (2007) Istambul Turkey 453-459.

3. Cairns R., Knew H. Y., Kenny M. J. The use of recycled rubber tyres in concrete construction. Sustainable Waste Management and Recycling. Thomas Telford Ltd, London (2004) 135-142.

4. Zhu X., Miao C., Liu J., Hong J. Influence of crumb rubber on frost resistance of concrete and effect mechanism. Procedia Engineering 27 (2012) 206-213.

5. Seemann R., Krause D., Numerical Modeling of Nomex Honeycomb Sandwich Cores at MesoScale Level. Composite Structures 159 (2016) 702-718.

6. Skarżyński Ł., Tejchman J., Calculations of fracture process zones on meso-scale in nothed concrete beams subjected to three point bending beams. European Journal of Mechanics A/Soils 29 (2010) 746-760.

7. Hangai Y., Takahashi K., Yamaguchia R., Utsunomiya T., Kitahara S., Kuwazuru O., Yoshikawa N. Nondestructive observation of pore structure deformation behavior of functionally graded aluminum foam by X-ray computed tomography. Materials Science and Engineering: A 55630 (2012) 678-684.

8. Liao K.Y., Chang P. K., Peng Y.N., Yang C.C. A Study on characteristics of interfacial transition zone in concrete. Cement and Concrete Research 34 (2004) 997-989.

9. Ye G., Percolation of capillary pores in hardening cement pastes. Cement and Concrete Research 35 (2005) 167- 176.

10. Tang T., Johnson D., Smith R. E., Felicelli S. D. Numerical evaluation of the temperature field of steady-state rolling tires. Applied Mathematical Modelling 38 (2014) 1622-1637.

11. He L., Ma Y., Liu Q., Mu Y. Surface modification of crumb rubber and its influence on the mechanical properties of rubber-cement concrete. Construction and Building Materials 120 (2016) 403-407.

12. Buyukozturk O. Interface fracture and crack propagation in concrete composites. C. Huet (Ed.), Micromechanics of Concrete and Cementitious Composites, Presses Polytechniques et Universitaires Romandes, Lausanne (1993) 203-212.

13. RILEM Draft Recommendations TC-89-FMT Determination of fracture parameters $K_{I c}$ and $C T O D_{c}$ of plan concrete using three point bending test Fracture Mechanics of Concrete Test Methods. Materials and Structures 23 (1990) 457-460.

14. Sadowski T., Pietras D. Description of degradation process of rubberized lean concrete. Solid State Phenomena 216 (2014) pp 67-72. 